\title{
A NOTE ON QUADRATIC RICCATI DIFFERENTIAL EQUATION USING PADÉ APPROXIMANT
}

\author{
Pedro Pablo Cárdenas Alzate *1四, Álvaro Andrés Quintero Orrego ${ }^{2}$ \\ ${ }^{* 1}$ Department of Mathematics, Universidad Tecnológica de Pereira and GEDNOL, Colombia \\ 2 Institución Universitaria EAM, Colombia
}

DOI: https://doi.org/10.29121/ijetmr.v7.i6.2020.697

Article Citation: Pedro Pablo Cárdenas Alzate, and Álvaro Andrés Quintero Orrego. (2020). A NOTE

ON QUADRATIC RICCATI

DIFFERENTIAL EQUATION USING

PADÉ APPROXIMANT. International Journal of Engineering Technologies and Management Research, 7(6),

$117-124$.

https://doi.org/10.29121/ijetmr.v7 i6.2020.697

Published Date: 20 June 2020

Keywords:

Padé Approximant

Quadratic Riccati

Semi-Analytical Method

Normal Padé table

\begin{abstract}
Semi-analytical methods for solving non-linear models require an initial approach to determine the solutions sought and the calculation of one or more fitting parameters. When the initial approach is chosen correctly, the results can be very precise, but not there is a general method for choosing such an initial approach. In this paper, it is suggested to use directly the serial solution of a non-linear model to find Padé's approximation with highly efficient results.
\end{abstract}

\section{INTRODUCTION}

The resolution of non-linear differential equations is a very important problem in the sciences in general since many phenomena are modeled using this type of equation. It is also true that in most cases, it is not possible to find analytical solutions to such models and therefore knowledge of efficient numerical methods to approximate them is essential. Thus, there are several semi-analytical methods that allow us to approximate the solutions numerically, such as the Adomian decomposition method, the differential transformation and the Padé method [1].

For this reason, the Padé Method is widely used in computer calculations. This method has proven to be very useful in obtaining quantitative information about the solution of many interesting problems in physics-mathematics and engineering. The applications of Padé's main approaches are divided into two classes:

- The provision of efficient rational approaches to special mathematical functions

- The acquisition of quantitative information about a function for which you only have qualitative information and coefficients in power series.

The Padé approximations, obtained as the quotient of two polynomials from Taylor's coefficients of series expansion, are the basis of many non-linear methods and have close connections with the famous $\varepsilon$-algorithm, continuous fractions and orthogonal polynomials. The Padé approximations are the non-linear counterpart of the first-order Taylor series expansions used in linear methods.

(C) 2020 The Author(s). This is an open access article distributed under the terms of the Creative Commons Attribution License, which permits unrestricted use, distribution, and reproduction in any medium, provided the original author and source are credited. 
A Note on Quadratic Riccati Differential Equation Using Padé Approximant

There are several methods to find Padé's approximations, several of them based on continuous fractions, so special attention is given to these fractions and their most important properties are illustrated.

\section{PADÉ APPROXIMANT METHOD}

\subsection{GENERALITIES OF THE PROPOSED METHOD}

The divergence of a power series is indicative of the presence of singularities. This divergence reflects then the inability of the polynomials to approximate a function around that singularity. The basic idea in sum theory is to represent $f(z)$, any function by a convergent expression. One of the methods used, which requires as input only a finite number of terms from a divergent series, is the sums of Padé [2].

In the technique proposed by Padé, the series of powers

$$
f(z)=\sum_{i=0}^{\infty} c_{i} z^{i}
$$

is replaced by a succession of rational functions of the form:

$$
R_{N}^{M}(z)=\frac{\sum_{i=0}^{M} a_{i} z^{i}}{\sum_{i=0}^{N} b_{i} z^{i}}
$$

Usually we take the standardization $b_{0}=1$ and the remaining $M+N+1$ coefficients $a_{0}, \cdots, a_{M}, b_{1, \cdots}, b_{N}$ in (2), are chosen in such a way that that match the $M+N+1$ coefficients in the power series expansion (1). That is,

$$
f(z)-R_{N}^{M}(z)=\mathcal{O}\left(z^{M+N+1}\right)
$$

From where

$$
f(z) \sum_{i=0}^{M} b_{i} z^{i}-\sum_{i=0}^{M} a_{i} z^{i}=\mathcal{O}\left(z^{M+N+1}\right)
$$

If $\sum a_{i} z^{i}$ is a serial representation of $f(z)$, then in many cases $R_{N}^{M}(z) \rightarrow f(z)$ when $M, N \rightarrow \infty$, even if $\sum a_{i} z^{i}$ is divergent. Generally, we consider the convergent succession $R_{0}^{J}, R_{1}^{1+J}, \cdots$, where $M=N+J$ with $J$ fixed and $N \rightarrow \infty$.

The $M+N+1$ coefficients are calculated as follows [3]:

Case 1: $M \geq N$

Developing the expression (4) we have:

$$
\begin{aligned}
& c_{0}+c_{1} z+c_{2} z^{2}+\ldots+\quad c_{N} z^{N}+\ldots+\quad c_{M} z^{M}+\ldots+\quad c_{M+N} z^{M+N} \\
& +c_{0} b_{1} z+c_{1} b_{1} z^{2}+\ldots+c_{N-1} b_{1} z^{N}+\ldots+c_{M-1} b_{1} z^{M}+\ldots+c_{M+N-1} b_{1} z^{M+N} \\
& +c_{0} b_{2} z^{2}+\ldots+c_{N-2} b_{2} z^{N}+\ldots+c_{M-2} b_{2} z^{M}+\ldots+c_{M+N-2} b_{2} z^{M+N} \\
& +c_{0} b_{N} z^{N}+\ldots+c_{M-N} b_{N} z^{M}+\ldots+\quad c_{M} b_{N} z^{M+N} \\
& =a_{0}+a_{1} z+a_{2} z^{2}+\ldots+a_{N} z^{N}+\ldots+\quad a_{M} z^{M}
\end{aligned}
$$

By equalizing coefficients in the above expression, we obtain the following systems: 
Pedro Pablo Cárdenas Alzate, and Álvaro Andrés Quintero Orrego

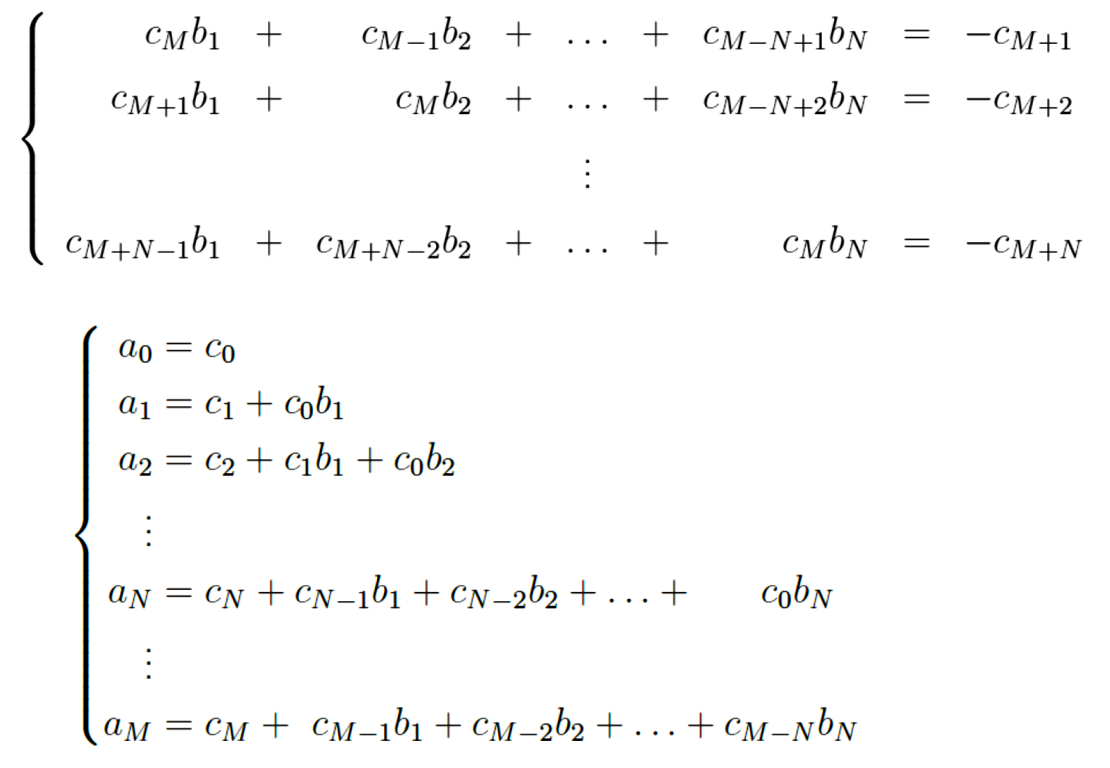

Case 2: $M<N$

$$
\begin{aligned}
& c_{0}+c_{1} z+c_{2} z^{2}+\ldots+\quad c_{M} z^{M}+\ldots+\quad c_{N} z^{N}+\ldots+\quad c_{M+N} z^{M+N} \\
& +c_{0} b_{1} z+c_{1} b_{1} z^{2}+\ldots+c_{M-1} b_{1} z^{M}+\ldots+c_{N-1} b_{1} z^{N}+\ldots+c_{M+N-1} b_{1} z^{M+N} \\
& \begin{array}{ccc}
+c_{0} b_{2} z^{2}+\ldots+c_{M-2} b_{2} z^{M}+\ldots+ & c_{N-2} b_{2} z^{N}+\ldots+c_{M+N-2} b_{2} z^{M+N} \\
\vdots & \vdots
\end{array} \\
& +c_{0} b_{M} z^{M}+\ldots+c_{N-M} b_{M} z^{N}+\ldots+\quad c_{N} b_{M} z^{M+N} \\
& +\quad c_{0} b_{N} z^{N}+\ldots+\quad c_{M} b_{N} z^{M+N} \\
& =a_{0}+a_{1} z+a_{2} z^{2}+\ldots+a_{M} z^{M}
\end{aligned}
$$

and after matching coefficients we get:

$$
\begin{aligned}
& \left\{\begin{array}{ccccc}
c_{M} b_{1}+ & c_{M-1} b_{2}+\ldots+ & c_{0} b_{M+1} & & =-c_{M+1} \\
c_{M+1} b_{1}+ & c_{M} b_{2}+\ldots+ & c_{1} b_{M+1}+ & c_{0} b_{M+2} & =-c_{M+2} \\
\vdots & & & \\
c_{N-1} b_{1}+ & c_{N-2} b_{2}+\ldots+c_{N-M-1} b_{M+1}+c_{N-M-2} b_{M+2}+\ldots+c_{0} b_{N} & =-c_{N} \\
\vdots & & & \\
c_{M+N-1} b_{1}+c_{M+N-2} b_{2}+\ldots+ & c_{N-1} b_{M+1}+ & c_{N-2} b_{M+2}+\ldots+c_{M} b_{N}=-c_{M+N}
\end{array}\right. \\
& \left\{\begin{array}{l}
a_{0}=c_{0} \\
a_{1}=c_{1}+c_{0} b_{1} \\
a_{2}=c_{2}+c_{1} b_{1}+c_{0} b_{2} \\
\quad \vdots \\
a_{M}=c_{M}+c_{M-1} b_{1}+c_{M-2} b_{2}+\ldots+c_{0} b_{M}
\end{array}\right.
\end{aligned}
$$


A Note on Quadratic Riccati Differential Equation Using Padé Approximant

By solving systems (5) and (7), we find the coefficients in the denominator of (2):

$$
\mathcal{A}\left[\begin{array}{c}
b_{1} \\
b_{2} \\
\vdots \\
b_{N}
\end{array}\right]=-\left[\begin{array}{c}
c_{M+1} \\
c_{M+2} \\
\vdots \\
c_{M+N}
\end{array}\right]
$$

Where $\mathrm{A}$ is a matrix $n \times n$ whose entries are given by $\mathrm{A}_{i j}=c_{M+i-j}$ with $a_{k}=0$ if $k<0$.

On the other hand, the coefficients $a_{0}, a_{1}, \cdots$ are determined from (6) and (8) with:

$$
a_{j}=\sum_{k=0}^{j} c_{j-k} b_{k}, \quad 0 \leq j \leq M
$$

Where $b_{k}=0$ for $K>N$. Here, the resulting rational function $R_{N}^{M}(z)$ is called a Padé approximation.

\subsection{PADÉ APPROXIMANT ALGORITHM}

Semi-analytical methods for solving non-linear models require an initial approach to determine the solutions sought and the calculation of one or more fitting parameters. When the initial approach is chosen correctly, the results can be very precise, but not there is a general method for choosing such an initial approach [4],[5]. In this paper, we suggest using directly the serial solution of a non-linear model to find Padé's approximation with highly efficient results.

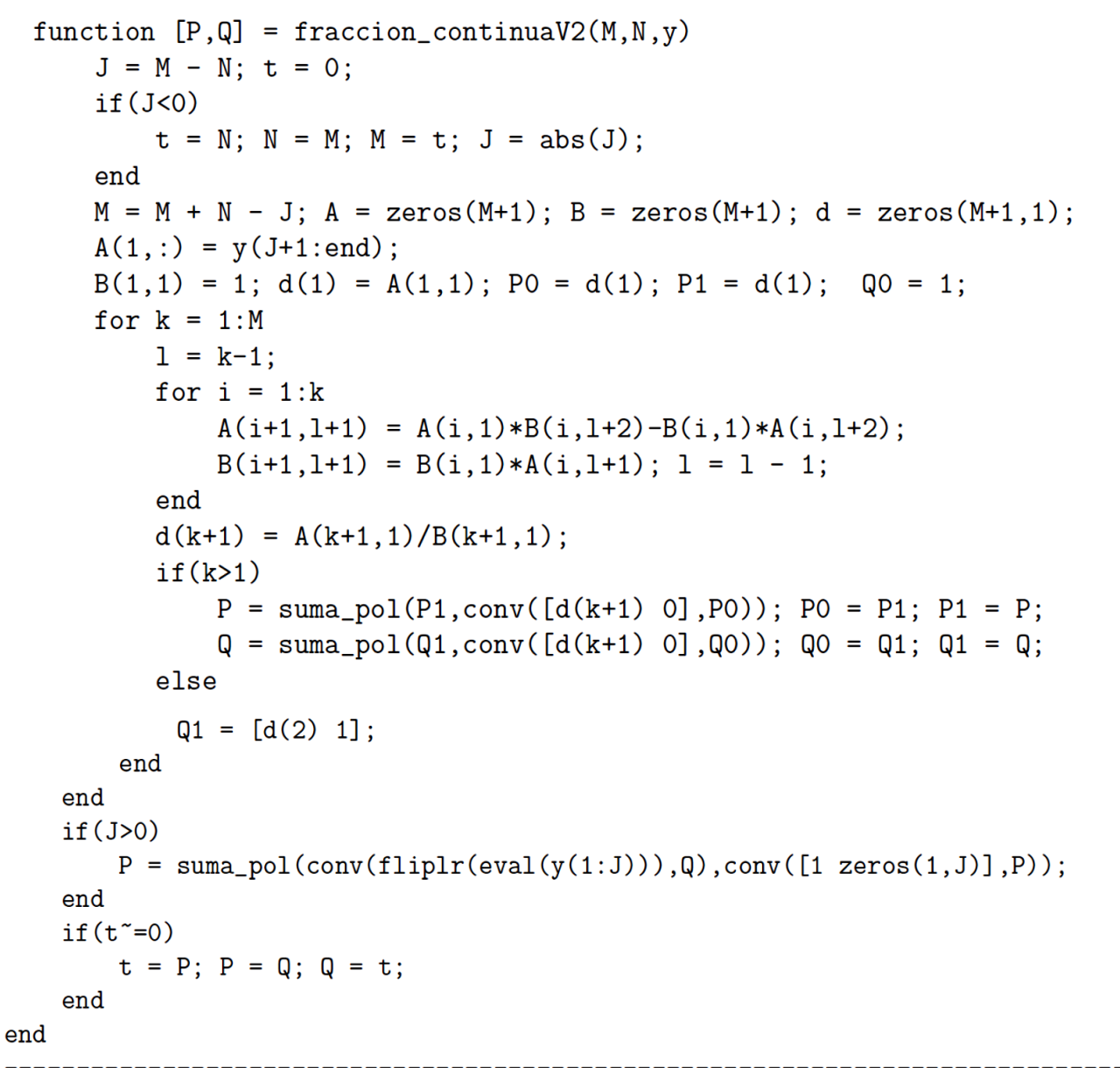


Pedro Pablo Cárdenas Alzate, and Álvaro Andrés Quintero Orrego

This algorithm receives as input parameters, in addition to the degrees of numerator and denominator of the Padé approximation, the coefficients of the serial expansion of the non-linear model solution instead of the explicit function.

\section{NUMERICAL RESULTS}

The Riccati quadratic equation is a well-known asymptotic problem with some degree of difficulty in solving by other approach techniques [6].

\subsection{NON-NORMAL PADÉ'S APPROACH}

Let us assume the model

$$
\left\{\begin{array}{c}
u^{\prime}-2 u+u^{2}-1=0 \\
u(0)=0
\end{array}\right.
$$

whose exact solution is given by

$$
u(x)=1+\sqrt{2}\left(\sqrt{2} x+\frac{1}{2} \ln \frac{\sqrt{2}-1}{\sqrt{2}+1}\right)
$$

Now, let us assume initially that

$u(x)=\sum_{n=0}^{\infty} c_{n} x^{n}$,

then

$$
\begin{aligned}
u^{\prime}-2 u+u^{2}-1 & =\sum_{n=0}^{\infty}(n+1) c_{n+1} x^{n}-2 \sum_{n=0}^{\infty} c_{n} x^{n}+\sum_{n=0}^{\infty}\left(\sum_{k=0}^{n} c_{k} c_{n-k}\right) x^{n}-1 \\
& =\sum_{n=0}^{\infty}\left[(n+1) c_{n+1}-2 c_{n}+\sum_{k=0}^{n} c_{k} c_{n-k}\right] x^{n}-1 \\
& =0
\end{aligned}
$$

From where, for $n=0$, we obtain

$$
c_{1}-2 c_{0}+c_{0}^{2}=1
$$

And for $n>0$ we have

$$
(n+1) c_{n+1}-2 c_{n}+\sum_{k=0}^{n} c_{k} c_{n-k}=0
$$

Therefore, as $u(0)=0$, then $c_{0}=0$.

However, if we consider the sequence $R_{0}^{0}, R_{1}^{1}, R_{2}^{2}, \ldots$ we can see that $R_{0}^{0}$ is not normal, in fact, $D_{0,0}=1, D_{1,0}=$ $1, D_{0,1}=c_{0}, D_{1,1}=c_{1}$ and as $c_{0}=0$, then $R_{0}^{0}$ is not normal (See Figure 1 ). 
A Note on Quadratic Riccati Differential Equation Using Padé Approximant

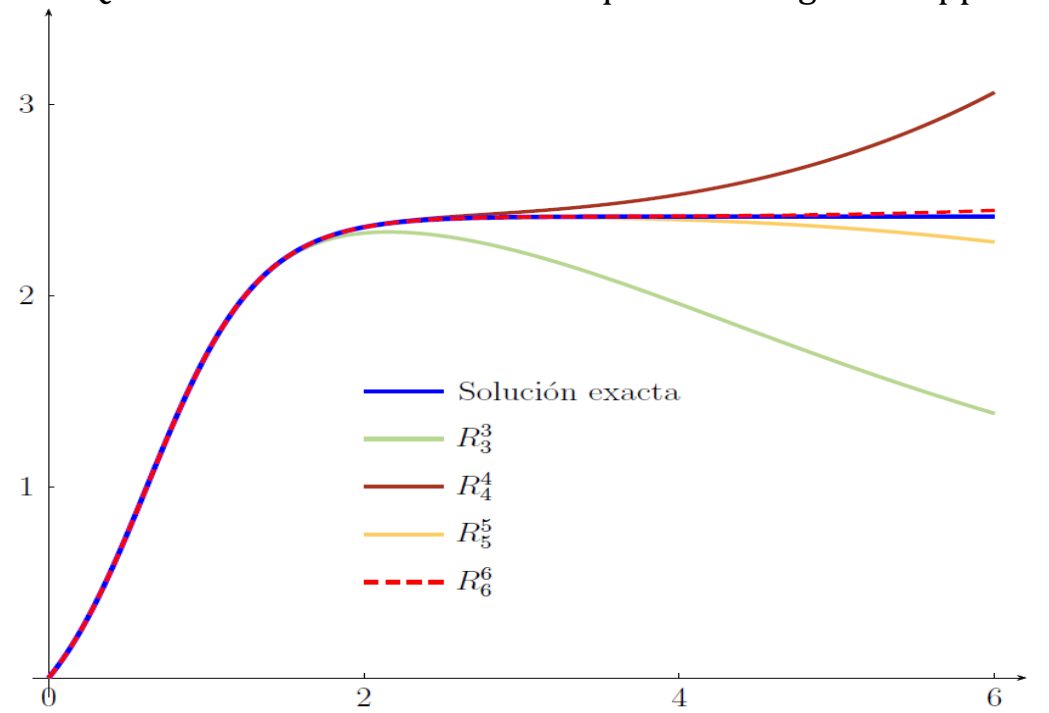

Figure 1: Exact solution and Padé's approximations of the model (1)

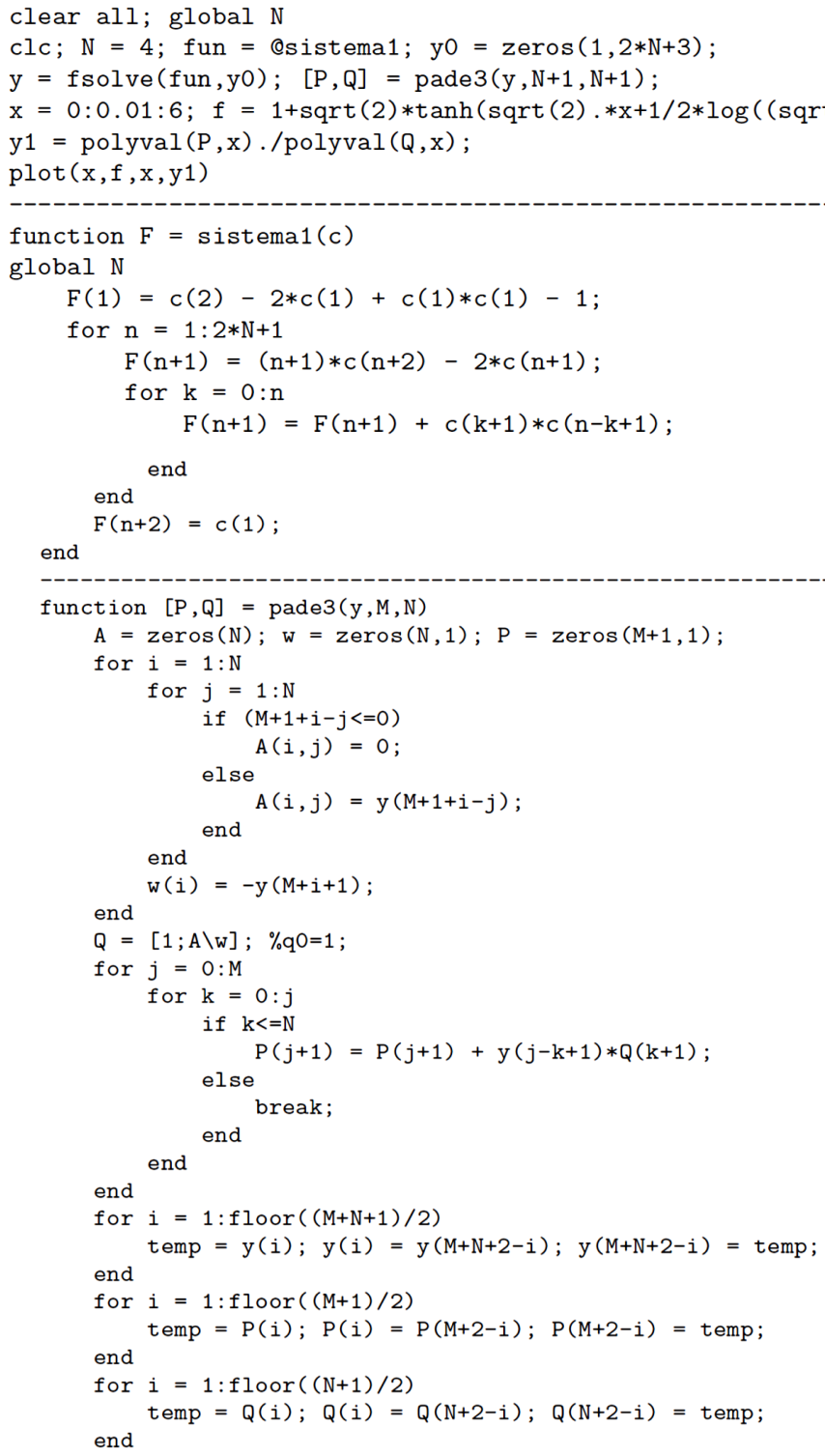




\subsection{NORMAL PADÉ'S APPROACH}

Pedro Pablo Cárdenas Alzate, and Álvaro Andrés Quintero Orrego

Let us assume the model

$$
\left\{\begin{array}{c}
u^{\prime}-2 u+u^{2}-1=0 \\
u(0)=1
\end{array}\right.
$$

Whose exact solution is given by

$$
u(x)=1+\sqrt{2} \tanh (\sqrt{2} x)
$$

In this case, as

$$
u(0)=1 \text {, }
$$

Then $c_{0}=1$. Now, we can see that the sequence $R_{0}^{0}, R_{1}^{0}, R_{1}^{1}, \ldots$ is normal, we use this algorithm to determine the Padé's approximant (See Figure 2).

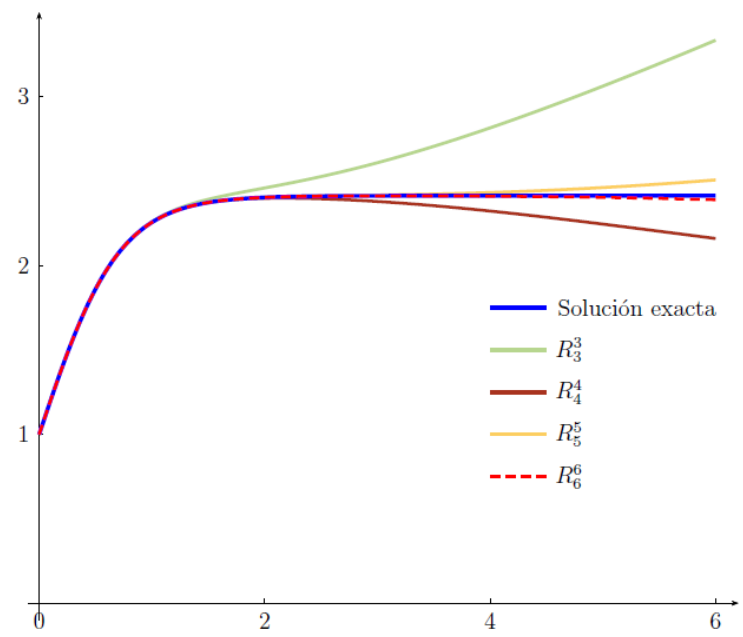

Figure 2: Exact solution and Padé's approximations of the model (2)

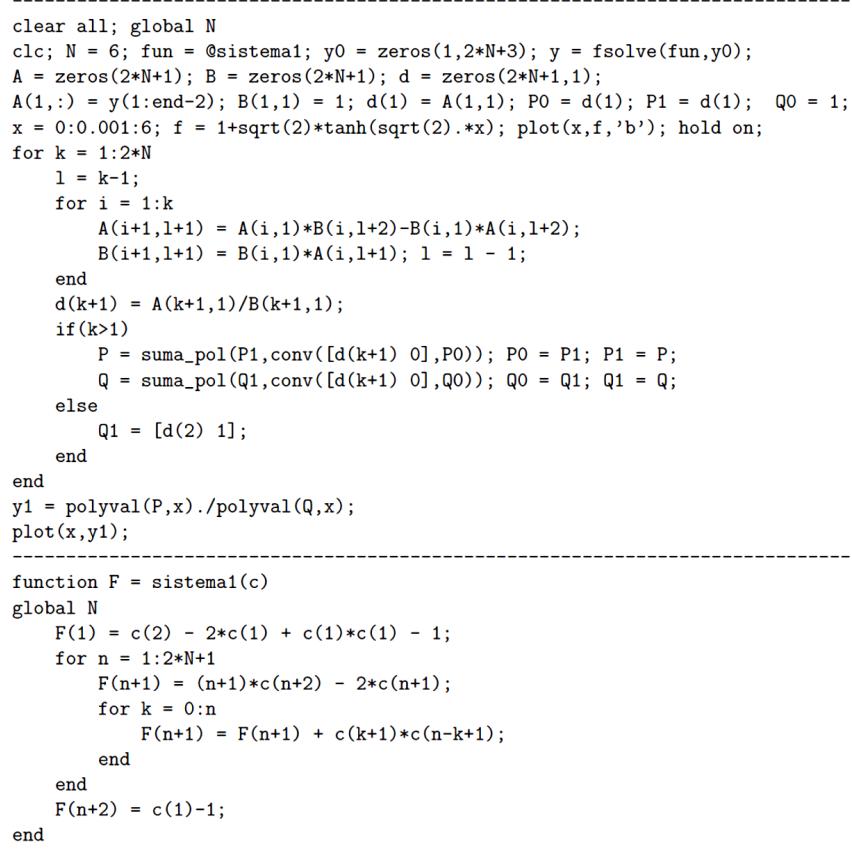




\section{CONCLUSION}

A Note on Quadratic Riccati Differential Equation Using Padé Approximant

In this work, we have illustrated the accuracy, simplicity and applicability of the Padé approach method used in different non-linear models. It was shown how Padé's approximations, calculated from the $M+N+1$ terms of Taylor's series expansion of a function, improve the approximation of this one with respect to the one resulting from truncation of the same Taylor series.

Two algorithms were carried out to determine the coefficients of the rational expression corresponding to the approximate of Padé of order $(M, N)$ : the first one by means of the solution of a system of linear equations for which the matrix of coefficients in a Toeplitz matrix very close to a singular matrix so solving the system may not be very efficient numerically; the second algorithm developed is much more efficient than the previous one because it uses the properties of continuous fractions to find Padé approximates of any order under the condition of normality of Padé's succession.

\section{SOURCES OF FUNDING}

None.

\section{CONFLICT OF INTEREST}

None.

\section{ACKNOWLEDGMENT}

The authors gratefully acknowledge the support of the Universidad Tecnológica de Pereira and the group GEDNOL and would like to thank the referee for his valuable suggestions that improved the presentation of the paper.

\section{REFERENCES}

[1] Padé, H. Sur la représentation approchée d'une fonction par des fractions rationnelles, Annales scientifiques de l'E.N.S. 3 série, tome 9 (1892), 3-93

[2] Padé, H. Mémoire sur les développements en fractions continues de la function exponentielle, pouvant servir d'introduction à la théorie des fractions continues algébraiques, Annales scientifiques de l'ENS. 3 série, tome 16 (1899), 395-426.

[3] Padé, H. Recherches sur la convergence des développements en fractions continues d'une certaine catégorie des fonctions. Annales scientifiques de l'ENS, 3 série, tome 24 (1907), 341-400.

[4] Shatnawi, M.T. Solving boundary layer problems by residual power series method. Journal of Mathematics Research, 8 (2016), 68-73.

[5] Cárdenas, P. An iterative method for solving two special cases of Lane-Emden type equations. American Journal of Computational Mathematics, 4(2014), 242-253.

[6] Vasquez, L et al. Direct application of Padé approximant for solving nonlinear differential equations. Springer Plus, 3(2014), 563-574. 